\title{
Article \\ A Chaotic Bio-Mechanics Model for the optimization of dairy industry
}

\author{
Meng Liu ${ }^{1,+}$, Kexin Meng ${ }^{2, \dagger}$,Shuli Mei ${ }^{3,+, * \mathbb{D}}$, and Ruyi Xing ${ }^{4,+}$ \\ 1 College of Information and Electrical Engineering, China Agricultural University; bynd_lm@163.com \\ 2 College of Information and Electrical Engineering, China Agricultural University; 17853516390@163.com \\ 3 College of Information and Electrical Engineering, China Agricultural University; meishuli@163.com \\ 4 Center of education technology, Heibei University of Engineering; xingruyi_hue@163.com \\ * Correspondence: meishuli@163.com \\ + These authors contributed equally to this work.
}

check for updates

Citation: Liu, M.; Meng, K.; Mei, S.; Xing R. A Chaotic Bio-Mechanics

Model. Preprints 2022, 1, 0.

https://doi.org/

Publisher's Note: MDPI stays neutral with regard to jurisdictional claims in published maps and institutional affiliations.

Copyright: (C) 2022 by the authors Licensee MDPI, Basel, Switzerland. This article is an open access article distributed under the terms and conditions of the Creative Commons Attribution (CC BY) license (https:// creativecommons.org/licenses/by/ $4.0 /)$.

\begin{abstract}
In the dairy industry a fundamental role is played by an efficient milk production, therefore even a slight shrinking in the production might have a significant consequence both on the industry profits and on the society. Milk is, in fact, one of the most common foods, and a fundamental support in the nutrition so that the lacking of milk should be avoided. An efficient milk production depends on various factors, but in particular it depends on the good health and supply of the dairy cow. However, in order to increase the milk production, cows are overfeed thus increasing their weight. Unfortunately, the overweight of the cows might easily damage their bones. In particular, the knee bones are fragile and they can easily swell and break. In order to analyze the knee bones and to compute a threshold leading to a damage, in this paper we study a bio-mechanical model of the knee joint and study the corresponding dynamical system. A novel dynamical model of dairy cow leg is constructed, in which the knee is represented by a complex structure of sliding and rolling. Then, the dynamic characteristics of the structure is analyzed by some numerical simulation. The results of the experiments shows that the solutions of the dynamical system might lead to a chaotic behavior. The evolution to chaos it explains the rising of various pathological features thus reducing the milk production.
\end{abstract}

Keywords: Dairy cow; Lyapunov exponent; bio-mechanical model; Nonlinear dynamics;gait; Weight scale; Three-link model; Fractals

\section{Introduction}

In order to get the highest yield and quality of milk for each cow with minimal investment, the farmers maximally exploit the dairy cows and feed them economically by preserving good health condition. The early symptoms of common cow diseases are often reflected in the changes of cow weight, body temperature, excitability, posture and gait. The changes of excitability and posture are reflected in the changes of gait. Changes in body weight and gait can cause changes of the signal curve obtained by the pressure sensor [1]. It is easy to know that the low-frequency component of the pressure sensor signal can be used to measure the weight of the dairy cow, and the high-frequency information in the compressive stress signal is able to reflect the changes of temperature, excitement and gait of dairy cows, which can be employed to analysis the early symptoms of common diseases of dairy cows[2]. In recent years, many researchers have found that there exists some bifurcation phenomenon in the dynamic system of cow legs [3]. Generally, the highfrequency component of the pressure sensor signal should be a chaotic signal and not a periodic measured from a healthy cow. On the contrary, to an unhealthy cow, such as a lame one, the chaotic phenomenon will be covered by the periodic component introduced by the periodic limp. Therefore, the chaotic dynamic leg model of the dairy cow is helpful to identify various unhealthy cow by means of a weighting system, which is showed in Figure 1. 


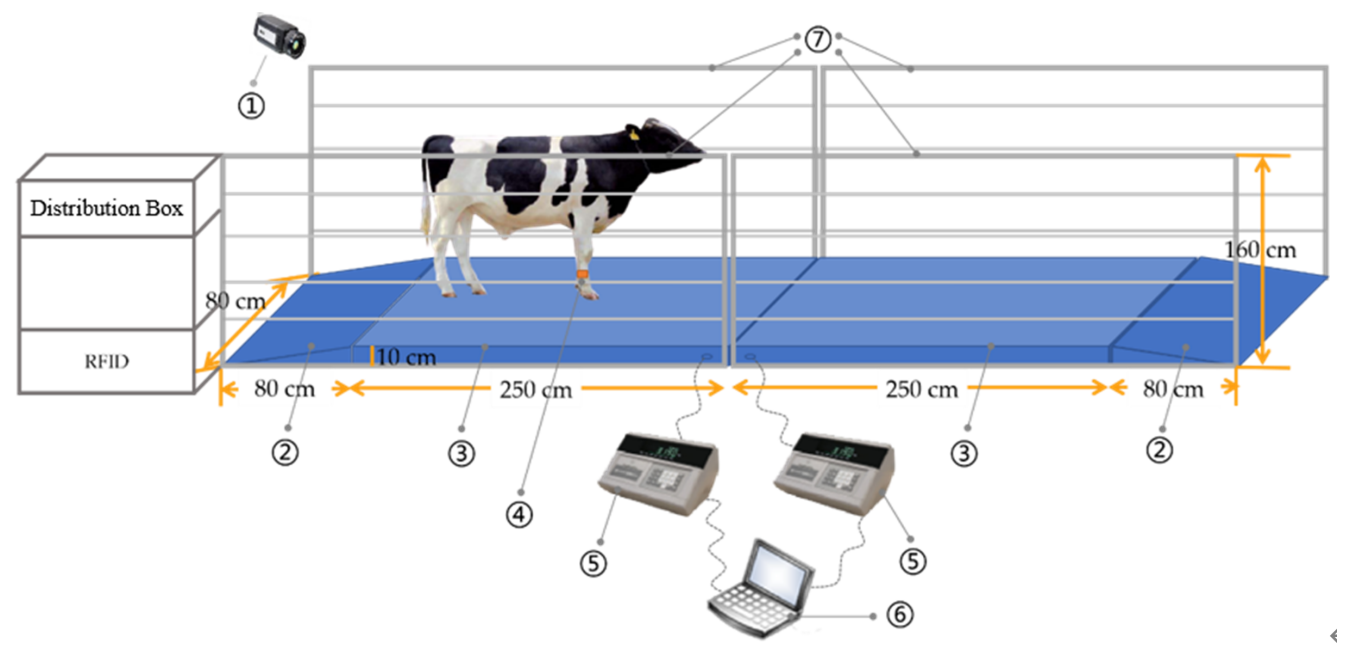

Figure 1. Pressure sensitive passageway

(1) Camera; (2) Slope; (3) Pressure sensors platform; (4) Anklet; (5) Pressure gauge; (6) Computer; (6) Fence.

Motivated by the widely development of the nonlinear dynamics in various physical fields. We try to construct a novel nonlinear dynamic model on the dairy cow leg, which can explain and identify the various walking postures of the cows with different activity and healthy. The model is sensitive to the initial angular velocity, that is, the different angular velocity can lead to more different Lyapunov exponents. Therefore, the exponents can be used to identify the different gait of the cow, which is helpful to detect the physiological indicators of dairy cows cheaply. In dynamical system theory, a phase space is a space in which all possible states of a system are represented, with each possible state corresponding to one unique point in the phase space. For mechanical systems, the phase space usually consists of all possible values of position and momentum variables. It is the outer product of direct space and reciprocal space. The concept of phase space was developed in the late 19th century by Ludwig Boltzmann, Henri Poincaré, and Josiah Willard Gibbs [4]. The phase diagramare useful tools in analyzing all kind of dynamical problems[5,6]. They are especially useful in studying the changes in motion as initial position and momentum are changed. Chaos theory is an interdisciplinary scientific theory and branch of mathematics focused on underlying patterns and deterministic laws highly sensitive to initial conditions in dynamical systems that were thought to have completely random states of disorder and irregularities [7]. Chaos theory has been widely used in many research and physical fields [8]. Styr, B., and I. Slutsky analyze the main types of possible homeostatic failures and provide the essential conceptual framework for examining the causal link between dysregulation of firing homeostasis, aberrant neural circuit activity and memory-related plasticity impairments associated with early $\mathrm{AD}$ [9]. In recent years, electromagnetic stimulation technology has been used to treat neurological diseases. Studies have shown that applying an external electric field to the nervous system can change the excitability of neurons, so as to achieve the purpose of curing neurological diseases. By applying external stimulation to neurons, neuronal membrane There are various discharge modes in the voltage, such as: resting state, cluster discharge and chaotic discharge $[10,11]$. 


\section{Construction of the chaotic dynamic system of cow leg}

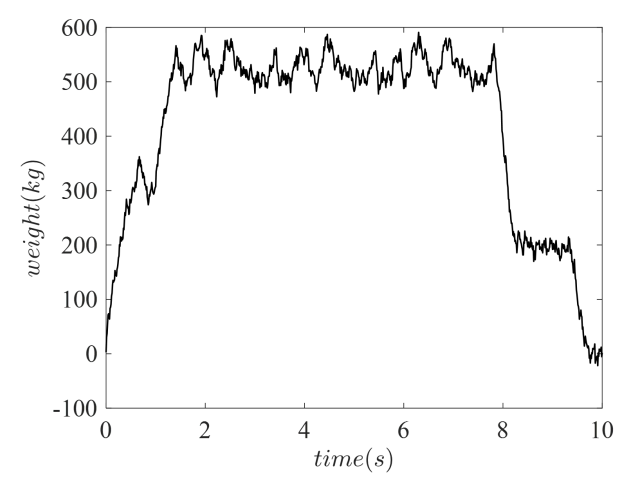

(a) Original signal

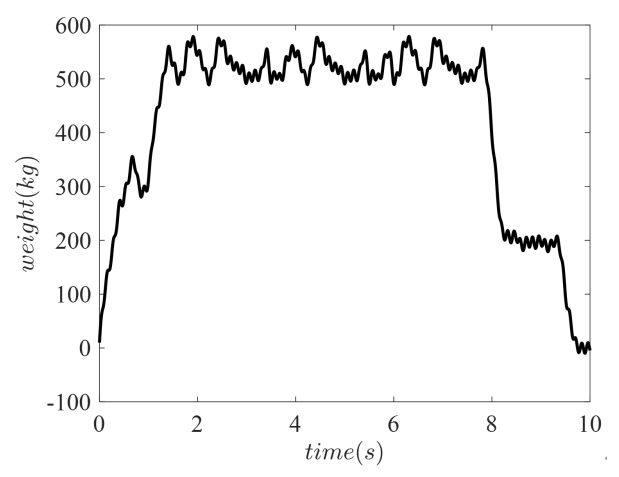

(b) Denoised signal

Figure 2. Cow's weight signal from stepping in to stepping out of the scale

The diary cow's weight signal from stepping in to stepping out of the scale is showed in Figure 4. Figure 2a is the original signa and Figure $2 a$ is the denoised signal. The left and right part of the figures denotes the processes of the cow stepping into and stepping out of the scale. In these two parts, there are only one or two legs standing on the scale. It is easy to find that there are many periodic signals with different frequencies are included in the weight signal, this reminds us that the dynamic system of the cow leg should not be described as a link model simply. In fact, the sliding structure of the knee has been taken into account in the design of modern prosthetic structures.

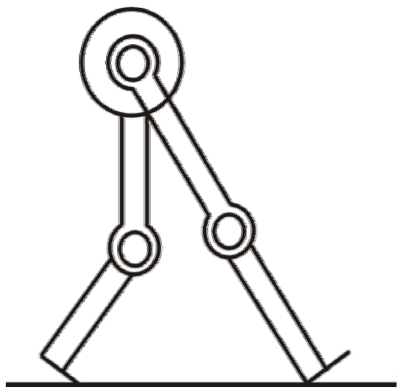

(a) Two-link model

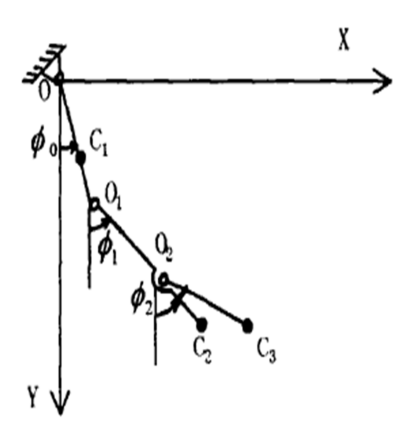

(b) three-link model

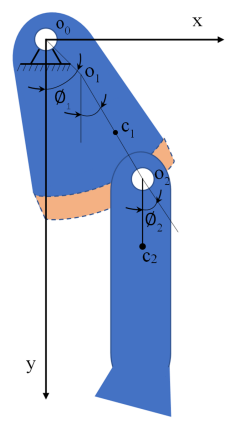

(c) Two-link with sliding model

Figure 3. Dynamic system of Cow Leg

In Figure 3, $m_{1}$ is the mass of eccentric $I$, the corresponding moment of inertia is $J_{C_{1}}=\frac{1}{4} m_{1} R_{1}^{2}$ to the centroid $C_{1}\left(x_{c_{1}}, y_{c_{1}}\right)$. The angular velocity of the eccentric $I$ is denoted as $\omega$, and and the eccentric distance and radius are denoted as $l_{1}$ and $R_{1}$, respectively.To the eccentric $I I, m_{2}$ is the mass, the moment of inertia is $J_{C_{2}}=\frac{1}{4} m_{2} R_{2}^{2}$ to the centroid $C_{2}\left(x_{c_{2}}, y_{c_{2}}\right)$.The velocities of the hinge $O_{2}\left(x_{o_{2}}, y_{o_{2}}\right)$ and the centroid $C_{2}$ are $V_{O_{2}}$ and $V_{C_{2}}$,respectively. the eccentric distance and radius are denoted as $l_{2}$ and $R_{2}$,respectively. Obviously, the above parameters satisfy the following relations:

$$
\begin{aligned}
x_{C_{1}}=l_{1} \sin \left(\phi_{0}\right) & \dot{x}_{C_{1}}=l_{1} \omega \cos \left(\phi_{0}\right) \\
y_{C_{1}}=l_{1} \cos \left(\phi_{0}\right) & \dot{y}_{C_{1}}=-l_{1} \omega \sin \left(\phi_{0}\right) \\
x_{O_{2}}=x_{C_{1}}+R_{1} \sin \left(\phi_{1}\right) & \dot{x}_{O_{2}}=\dot{x}_{C_{1}}+R_{1} \dot{\phi}_{1} \cos \left(\dot{\phi}_{1}\right) \\
y_{O_{2}}=y_{C_{1}}+R_{1} \cos \left(\phi_{1}\right) & \dot{y}_{O_{2}}=\dot{y}_{C_{1}}-R_{1} \dot{\phi}_{1} \sin \left(\phi_{1}\right) \\
x_{C_{2}}=x_{O_{2}}+l_{2} \sin \left(\phi_{2}\right) & \dot{x}_{C_{2}}=\dot{x}_{O_{2}}+l_{2} \dot{\phi}_{2} \cos \left(\phi_{2}\right) \\
y_{C_{2}}=y_{O_{2}}+l_{2} \cos \left(\phi_{2}\right) & \dot{y}_{C_{2}}=\dot{y}_{O_{2}}-l_{2} \dot{\phi}_{2} \sin \left(\phi_{2}\right)
\end{aligned}
$$

To the cow leg dynamic system, the different initial angular velocity can lead to different phase space trajectory, and our purpose is to test the system response is sensitive to the 
initial condition, so, the angular velocity can be assumed as a constant for simplify, that is, $\phi=\omega t$.

The velocity of the hinge $\mathrm{O}_{2}$ is defined as

$$
\begin{aligned}
V_{O_{2}}^{2} & =\left(\dot{x}_{O_{2}}\right)^{2}+\left(\dot{y}_{O_{2}}\right)^{2} \\
& =\left(\dot{x}_{C_{1}}+R_{1} \dot{\phi}_{1} \cos \left(\phi_{1}\right)\right)^{2}+\left(\dot{y}_{C_{1}}-R_{1} \dot{\phi}_{1} \sin \left(\phi_{1}\right)\right)^{2} \\
& =\left(l_{1} \omega\right)^{2}+\left(R_{1} \dot{\phi}_{1}\right)^{2}+2 \dot{\phi}_{1} l_{1}\left(\dot{x}_{C_{1}} \cos \left(\phi_{1}\right)-\dot{y}_{C_{1}} \sin \left(\phi_{1}\right)\right) \\
& =\left(l_{1} \omega\right)^{2}+\left(R_{1} \dot{\phi}_{1}\right)^{2}+2 \dot{\phi}_{1} l_{1} l_{2} \omega \cos \left(\omega t-\phi_{1}\right)
\end{aligned}
$$

The velocity of the centroid $C_{2}$

$$
\begin{aligned}
V_{C_{2}}^{2}= & \left(\dot{x}_{C_{2}}\right)^{2}+\left(\dot{y}_{C_{2}}\right)^{2} \\
= & \left(l_{1} \omega \cos \left(\phi_{0}\right)+R_{1} \dot{\phi}_{1} \cos \left(\phi_{1}\right)+l_{2} \dot{\phi}_{2} \cos \left(\phi_{2}\right)\right)^{2}- \\
& \left(l_{1} \omega \sin \left(\phi_{0}\right)+R_{1} \dot{\phi}_{1} \sin \left(\phi_{1}\right)+l_{2} \dot{\phi}_{2} \sin \left(\phi_{2}\right)\right)^{2} \\
= & \left(V_{O_{2}}\right)^{2}+\left(l_{2} \dot{\phi}_{2}\right)^{2}+2 l_{1} l_{2} \dot{\phi}_{2} \omega \sin \left(\phi_{0}-\phi_{2}\right)+2 R_{1} l_{2} \dot{\phi}_{1} \dot{\phi}_{2} \omega \cos \left(\phi_{0}-\phi_{2}\right)
\end{aligned}
$$

The kinetic energy of the system can be calculated as follows

1. The eccentric wheel $I$ rotates as a fixed axis, the kinetic energy is

$$
T_{1}=\frac{1}{2} m_{1}\left(l_{1} \omega\right)^{2}+\frac{1}{2} J_{C_{1}} \omega^{2}=\frac{1}{8} m_{1} \omega^{2}\left(4 l_{1}^{2}+R_{1}^{2}\right)
$$

2. The eccentric wheel II do the plane movement, the kinetic energy is

$$
\begin{aligned}
T_{2}= & \frac{1}{2} m_{2}\left(V_{C_{2}}\right)^{2}+\frac{1}{2} J_{C_{2}}\left(\dot{\phi}_{2}\right)^{2} \\
= & \frac{1}{2} m_{2}\left[\left(l_{1} \omega\right)^{2}+\left(R_{1} \dot{\phi}_{1}\right)^{2}+2 \dot{\phi}_{1} l_{1} l_{2} \omega \cos \left(\omega t-\phi_{1}\right)+\left(l_{2} \dot{\phi}_{2}\right)^{2}+\right. \\
& \left.2 \dot{\phi}_{2} l_{1} l_{2} \omega \sin \left(\phi_{0}-\phi_{2}\right)\right]+\frac{1}{8} m_{2}\left(R_{2} \dot{\phi}_{2}\right)^{2}
\end{aligned}
$$

The Total kinetic energy is defined as

$$
\begin{aligned}
T= & T_{1}+T_{2} \\
= & \frac{1}{2} m_{2}\left[\left(l_{1} \omega\right)^{2}+\left(R_{1} \dot{\phi}_{1}\right)^{2}+2 \dot{\phi}_{1} l_{1} l_{2} \omega \cos \left(\omega t-\phi_{1}\right)+\right. \\
& \left.\left(l_{2} \dot{\phi}_{2}\right)^{2}+2 \dot{\phi}_{2} l_{1} l_{2} \omega \sin \left(\phi_{0}-\phi_{2}\right)+2 R_{1} l_{2} \dot{\phi}_{1} \dot{\phi}_{2} \cos \left(\phi_{1}-\phi_{2}\right)\right]+ \\
& \frac{1}{8}\left[m_{1} \omega^{2}\left(4 l_{1}^{2}+R_{1}^{2}\right)+m_{2}\left(R_{2} \dot{\phi}_{2}\right)^{2}\right]
\end{aligned}
$$

Next, we calculate the potential energy of the system.

In case $\phi_{0}=\phi_{1}=\phi_{2}=0$, the potential energy of the system is 0 , in other cases, the potential energy of the system is

$$
U_{1}=m_{1} g l_{1}(1-\cos (\omega t))
$$

The potential energy of the eccentric wheel $I I$ is

$$
U_{2}=m_{2} g\left[l_{1}(1-\cos (\omega t))+R_{1}\left(1-\cos \left(\phi_{1}\right)\right)+l_{2}\left(1-\cos \left(\phi_{2}\right)\right)\right]
$$


The total potential energy of the system is

$$
\begin{aligned}
U= & U_{1}+U_{2} \\
= & m_{1} g l_{1}(1-\cos (\omega t))+ \\
& m_{2} g\left[l_{1}(1-\cos (\omega t))+R_{1}\left(1-\cos \left(\phi_{1}\right)\right)+l_{2}\left(1-\cos \left(\phi_{2}\right)\right)\right] \\
= & \left(m_{1}+m_{2}\right) g l_{1}(1-\cos (\omega t))+m_{2} g\left[R_{1}\left(1-\cos \left(\phi_{1}\right)\right)+l_{2}\left(1-\cos \left(\phi_{2}\right)\right)\right]
\end{aligned}
$$

According to definition of the Laplace function, we have

$$
\begin{aligned}
L= & T-U \\
= & \frac{1}{2} m_{2}\left[\left(l_{1} \omega\right)^{2}+\left(R_{1} \dot{\phi}_{1}\right)^{2}+2 \dot{\phi}_{1} l_{1} l_{2} \omega \cos \left(\omega t-\phi_{1}\right)+\right. \\
& \left.\left(l_{2} \dot{\phi}_{2}\right)^{2}+2 \dot{\phi}_{2} l_{1} l_{2} \omega \sin \left(\phi_{0}-\phi_{2}\right)+2 R_{1} l_{2} \dot{\phi}_{1} \dot{\phi}_{2} \cos \left(\phi_{1}-\phi_{2}\right)\right]
\end{aligned}
$$

Take the Laplace function Equation(5) into the second kind of Lagrange equation, we obtain

$$
\frac{\mathrm{d}}{\mathrm{d} t}\left(\frac{\partial L}{\partial \dot{\phi}_{i}}\right)-\frac{\partial L}{\partial \phi_{i}}=0
$$

where $i=1,2$.

$$
\begin{aligned}
\frac{\partial L}{\partial \dot{\phi}_{1}}= & m_{2}\left(R_{1}\right)^{2} \dot{\phi}_{1}+m_{2} l_{1} R_{1} \omega \cos \left(\omega t-\phi_{1}\right)+m_{2} R_{1} l_{2} \dot{\phi}_{2} \cos \left(\phi_{1}-\phi_{2}\right) \\
\frac{\partial L}{\partial \dot{\phi}_{2}}= & \frac{1}{4} m_{2} \dot{\phi}_{2}\left(\left(2 l_{2}\right)^{2}+\left(R_{2}\right)^{2}\right)+m_{2} l_{1} l_{2} \omega \sin \left(\omega t-\phi_{2}\right)+m_{2} R_{1} l_{2} \dot{\phi}_{1} \cos \left(\phi_{1}-\phi_{2}\right) \\
\frac{\mathrm{d}}{\mathrm{d} t}\left(\frac{\partial L}{\partial \dot{\phi}_{1}}\right)= & m_{2}\left(R_{1}\right)^{2} \ddot{\phi}_{1}-m_{2} l_{1} R_{1} \omega\left(\omega-\dot{\phi}_{1}\right) \sin \left(\omega t-\phi_{1}\right)+ \\
& m_{2} R_{1} l_{2} \ddot{\phi}_{2} \cos \left(\phi_{1}-\phi_{2}\right)-m_{2} R_{1} l_{2} \dot{\phi}_{2}\left(\dot{\phi}_{1}-\dot{\phi}_{2}\right) \sin \left(\phi_{1}-\phi_{2}\right) \\
\frac{\mathrm{d}}{\mathrm{d} t}\left(\frac{\partial L}{\partial \dot{\phi}_{2}}\right)= & \frac{1}{4} m_{2} \ddot{\phi}_{2}\left(\left(2 l_{2}\right)^{2}+\left(R_{2}\right)^{2}\right)+m_{2}\left(R_{1}\right)^{2} \ddot{\phi}_{1}- \\
& m_{2} l_{1} l_{2} \omega\left(\omega-\dot{\phi}_{2}\right) \cos \left(\omega t-\phi_{2}\right)+ \\
& m_{2} R_{1} l_{2} \ddot{\phi}_{1} \cos \left(\phi_{1}-\phi_{2}\right)-m_{2} R_{1} l_{2} \dot{\phi}_{1}\left(\dot{\phi}_{1}-\dot{\phi}_{2}\right) \sin \left(\phi_{1}-\phi_{2}\right) \\
\frac{\partial L}{\partial \phi_{1}}= & -m_{2} g R_{1} \sin \left(\phi_{1}\right)+m_{2} l_{1} R_{1} \omega \dot{\phi}_{1} \sin \left(\omega t-\phi_{1}\right)-m_{2} l_{2} R_{1} \dot{\phi}_{1} \dot{\phi}_{2} \sin \left(\phi_{1}-\phi_{2}\right) \\
\frac{\partial L}{\partial \phi_{2}}= & -m_{2} g l_{2} \sin \left(\phi_{2}\right)-m_{2} l_{1} l_{2} \omega \dot{\phi}_{2} \cos \left(\omega t-\phi_{2}\right)+m_{2} l_{2} R_{1} \dot{\phi}_{1} \dot{\phi}_{2} \sin \left(\phi_{1}-\phi_{2}\right)
\end{aligned}
$$

Thus, the second kind of Lagrange equation can be rewritten as the nonlinear system of ordinary differential equations as follows

$$
\left[\begin{array}{ll}
a_{11} & a_{12} \\
a_{21} & a_{22}
\end{array}\right]\left\{\begin{array}{l}
\ddot{\phi}_{1} \\
\ddot{\phi}_{2}
\end{array}\right\}=\left\{\begin{array}{l}
b_{1} \\
b_{2}
\end{array}\right\}
$$


where,

$$
\begin{aligned}
a_{11}= & m_{2}\left(R_{1}\right)^{2} \\
a_{12}= & m_{2} l_{2} R_{1} \cos \left(\phi_{1}-\phi_{2}\right) \\
a_{21}= & a_{12} \\
a_{22}= & \frac{1}{4} m_{2}\left(\left(2 l_{2}\right)^{2}+\left(R_{2}\right)^{2}\right) \\
b_{1}= & m_{2} l_{1} R_{1} \omega\left(\omega-\dot{\phi}_{1}\right) \sin \left(\omega t-\phi_{1}\right)+m_{2} l_{2} R_{1} \omega \dot{\phi}_{2} \sin \left(\phi_{1}-\phi_{2}\right)+ \\
& m_{2} l_{1} R_{1} \omega \dot{\phi}_{1} \sin \left(\omega t-\phi_{1}\right)-m_{2} l_{2} R_{1} \dot{\phi}_{1} \dot{\phi}_{2} \sin \left(\phi_{1}-\phi_{2}\right)-m_{2} g R_{1} \sin \left(\phi_{1}\right) \\
= & m_{2} l_{1} R_{1} \omega^{2} \sin \left(\omega t-\phi_{1}\right)-m_{2} l_{2} R_{1}\left(\dot{\phi}_{2}\right)^{2} \sin \left(\phi_{1}-\phi_{2}\right)-m_{2} g R_{1} \sin \left(\phi_{1}\right) \\
b_{2}= & -m_{2} l_{1} l_{2} \omega\left(\omega-\dot{\phi}_{2}\right) \cos \left(\omega t-\phi_{2}\right)-m_{2} l_{1} l_{2} \omega \dot{\phi}_{2} \cos \left(\omega t-\phi_{2}\right)+ \\
& m_{2} l_{1} R_{1} \dot{\phi}_{1}\left(\dot{\phi}_{1}-\dot{\phi}_{2}\right) \sin \left(\phi_{1}-\phi_{2}\right)+m_{2} l_{2} R_{1} \dot{\phi}_{1} \dot{\phi}_{2} \sin \left(\phi_{1}-\phi_{2}\right)-m_{2} g l_{2} \sin \left(\phi_{2}\right) \\
= & -m_{2} l_{2} R_{1} \omega^{2} \cos \left(\omega t-\phi_{2}\right)+m_{2} l_{2} R_{1}\left(\dot{\phi}_{1}\right)^{2} \sin \left(\phi_{1}-\phi_{2}\right)-m_{2} g l_{2} \sin \left(\phi_{2}\right)
\end{aligned}
$$

Eq.(7) is the dynamic system of the proposed model of dairy cow leg.

\section{Dynamical simulation experiments}

Qualitative and quantitative methods are usually used to judge whether the system vibration has chaotic components. In the qualitative method, the phase diagram of nonlinear dynamic model is often taken as the judge tools. The phase diagram of chaotic vibration system has the characteristics of non-repetition, irregularity and repeated winding. The typical characterization method is by mean of the Lyapunov exponent, it is well known that the system can produce the chaotic vibration as the maximum of the Lyapunov exponent is greater than $0[14-16]$.

\subsection{Phase diagram method}

For the convenience of calculation, We first give the calculation formula required for the phase diagram. Let

$$
\mathbf{A}=\left|\begin{array}{ll}
a_{11} & a_{12} \\
a_{21} & a_{22}
\end{array}\right| \quad \mathbf{B}_{1}=\left|\begin{array}{ll}
b_{1} & a_{12} \\
b_{2} & a_{22}
\end{array}\right| \quad \mathbf{B}_{2}=\left|\begin{array}{ll}
a_{11} & b_{1} \\
a_{21} & b_{2}
\end{array}\right|
$$

Then, the solution of the system of Eq.(7) can be expressed as

$$
\ddot{\phi}_{1}=\mathbf{B}_{1} / \mathbf{A} \quad \ddot{\phi}_{2}=\mathbf{B}_{2} / \mathbf{A}
$$

And then, let $y_{1}=\phi_{1}, y_{2}=\phi_{2}, y_{3}=\dot{\phi}_{1}, y_{4}=\dot{\phi}_{2}, f_{3}(\mathbf{y})=\mathbf{B}_{1}, f_{4}(\mathbf{y})=\mathbf{B}_{2}$,where, $\mathbf{y}=\left[y_{1}, y_{2}, y_{3}, y_{4}\right]^{T}$.Substituting above expressions into Eq.(7), the state-space model of Eq.(7) can be obtained as

$$
\left\{\begin{array}{l}
\dot{y}_{1}=y_{3} \\
\dot{y}_{2}=y_{4} \\
\dot{y}_{3}=f_{3}(\mathbf{y}) \\
\dot{y}_{4}=f_{4}(\mathbf{y})
\end{array}\right.
$$

The phase diagram can be plotted based on the vectors $\left(y_{1}, y_{3}\right)$ and $\left(y_{2}, y_{4}\right)$, which are the 2-D lines plot of the data in $y_{3}$ and $y_{3}$ versus the corresponding values in $y_{1}$ and $y_{2}$, respectively.

\subsection{Calculation of the maximum of the Lyapunov exponent $\lambda_{\text {max }}$}

There are many methods to calculate the Lyapunov exponent $\lambda$, such as the calculation method by means the definition of the Lyapunov exponent, Orthogonalization method 
, Wolf method, and so on. Here we take the definition method to calculate it. Let $\mathbf{f}=$ $\left[f_{1} f_{2} f_{3} f_{4}\right]^{T}, \mathbf{f} \subset C^{1}$, the Jacobi matrix of $\mathrm{f}$ with respect to the vector $\mathrm{y}$ can be expressed as

$$
\mathbf{J}=\left.\frac{\partial \mathbf{f}(\mathbf{y})}{\partial \mathbf{y}}\right|_{\mathbf{y}=\mathbf{y}(t)}=\left|\begin{array}{cccc}
0 & 0 & 1 & 0 \\
0 & 0 & 0 & 1 \\
\frac{\partial f_{3}}{\partial f_{1}} & \frac{\partial f_{3}}{\partial f_{2}} & \frac{\partial f_{3}}{\partial f_{3}} & \frac{\partial f_{3}}{\partial f_{4}} \\
\frac{\partial f_{4}}{\partial f_{1}} & \frac{\partial f_{4}}{\partial f_{2}} & \frac{\partial f_{4}}{\partial f_{3}} & \frac{\partial f_{4}}{\partial f_{4}}
\end{array}\right|
$$

where, $f_{1}(\mathbf{y})=y_{3}, f_{2}(\mathbf{y})=y_{4}$.To the state space equation (8), the value of the thee tangent vector $\delta \mathbf{y}(t)$ with respect to the parameter $t$ is determined by the Linearized form of Eq.(8) as follows

$$
\delta \dot{\mathbf{y}}(t)=\mathbf{J} \delta \mathbf{y}(t)
$$

Let $\delta y_{i}\left(t_{0}\right), i=1,2, \ldots, n$, is the initial condition of the Jacobi matrix [9], based on the definition of the Lyapunov exponent, we have

$$
\lambda_{i}=\lim _{t \rightarrow \infty} \frac{1}{t} \ln \left|\frac{\delta \mathbf{y}(t)}{\delta y_{i}\left(t_{0}\right)}\right|
$$

The Lyapunov exponent is a quantitative expression of the system's sensitivity to initial conditions and is an average of the long-term behavior of the system's local contraction or divergence. An $n$-dimensional dynamical system has $n$ Lyapunov exponents. A positive Lyapunov exponent is a measure of the degree to which the average exponents of two adjacent orbitals diverge; a negative Lyapunov exponent is a measure of the degree to which the average exponents of two adjacent orbitals converge. The sum of the Lyapunov exponents is the average of the volume change rates. Therefore, a dissipative system has at least one negative exponent, and the sum of the Lyapunov exponents is negative. The maximum Lyapunov exponent of a chaotic system is generally positive. The calculation procedure can be described as the Table 1.

Table 1. Calculation procedure of the Lyapunov

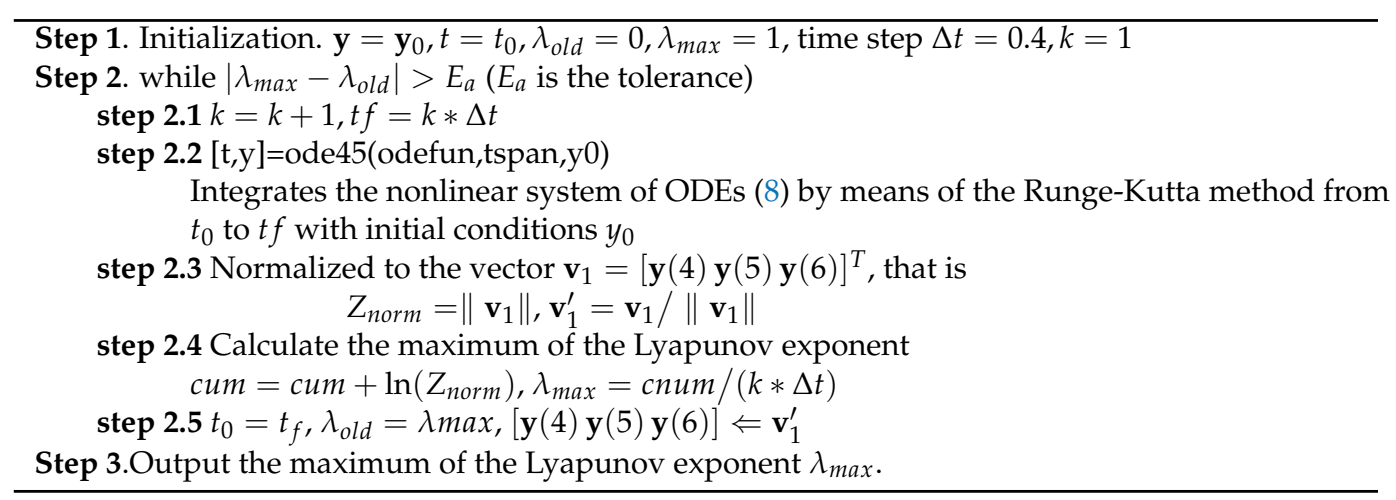

\section{Results and Discussion}

\subsection{Phase diagram}

In simulation, the masses of the two cams are $m_{1}=60, m_{2}=60$,respectively; $l_{0}=$ $0.0003 ; l_{1}=1.0003 ; l_{2}=0.02 ; R=0.02$.The phase diagram of the proposed model are showed in Figs.4 $(\mathrm{a} \sim \mathrm{c})$. With the increase of the angular velocity of the driving wheel, the phase diagram of the system becomes more and more complex, and the chaotic components become more and more. To some extent, it also reflects the process of the system from periodic motion to chaos. This illustrates the proposed method can be employed to identify the cow's gait in various physiological state.

The three-link model is the common model used to describe the cow legs.In Ref.[12],Long believed that the model is also a kind of chaos system. In numerical experiments, we take 
the same initial conditions as that appeared in Ref.[12],that is, $m_{1}=120.6593, m_{2}=11.7621$; $l_{0}=0.00039 ; l_{1}=1.0002 ; l_{2}=0.002 ; R=0.02$. The phase diagrams of the three-link model with different angular velocities are showed in Fig. $4(\mathrm{~d})$. In the case $\omega=2 \pi$, the phase diagrams showed in Fig.4(e) are not coincided exactly. In case $\omega=10 \pi$, the phase diagrams are almost exactly coincided. The diagram in the middle of the Fig.4(e) is the detail view of the part domain of left one in red rectangle. This means that the three-link system is almost a simple harmonic vibration system, and so it is difficult to identify the cow's health states exactly.

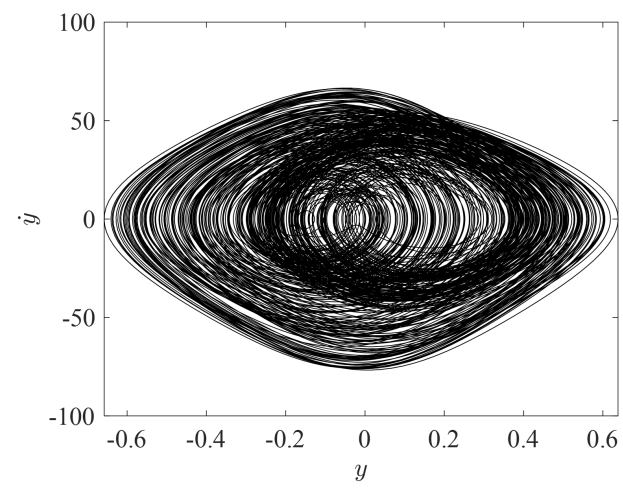

(a) $\omega=20 \pi$

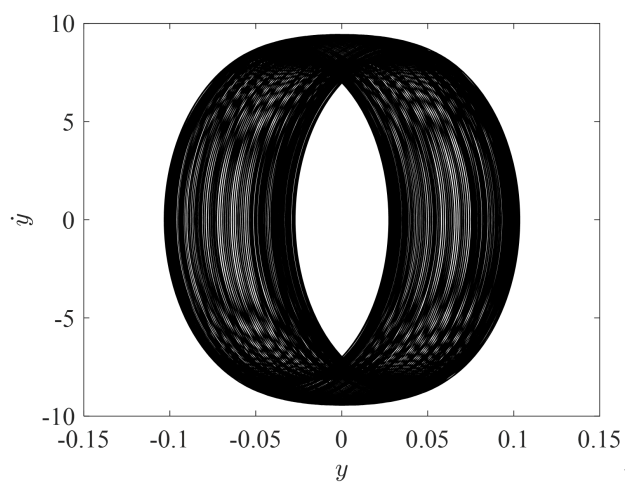

(c) $\omega=2 \pi$

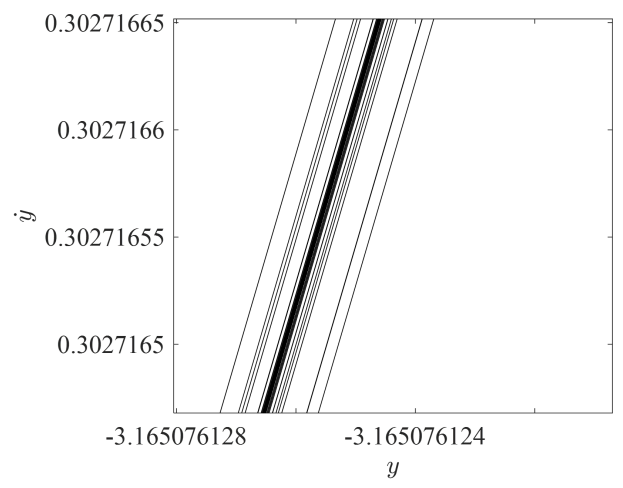

(e) $\omega=10 \pi$

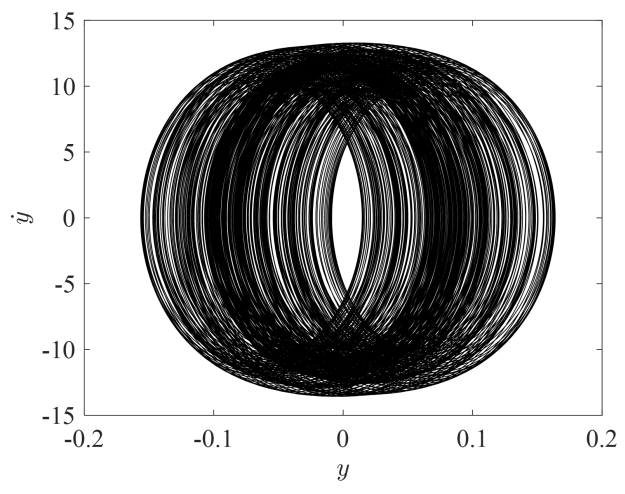

(b) $\omega=10 \pi$

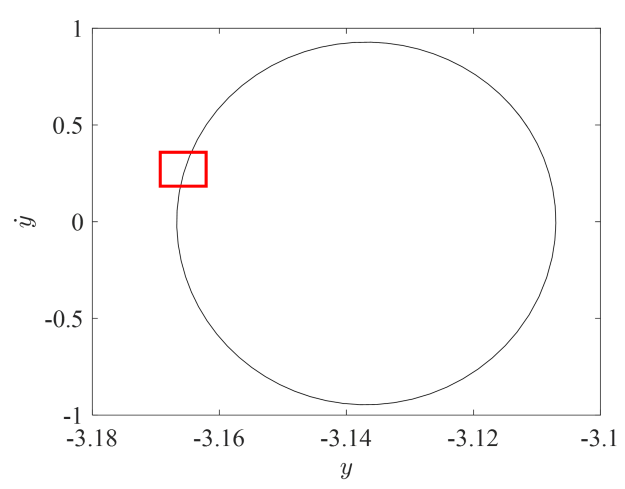

(d) $\omega=10 \pi$

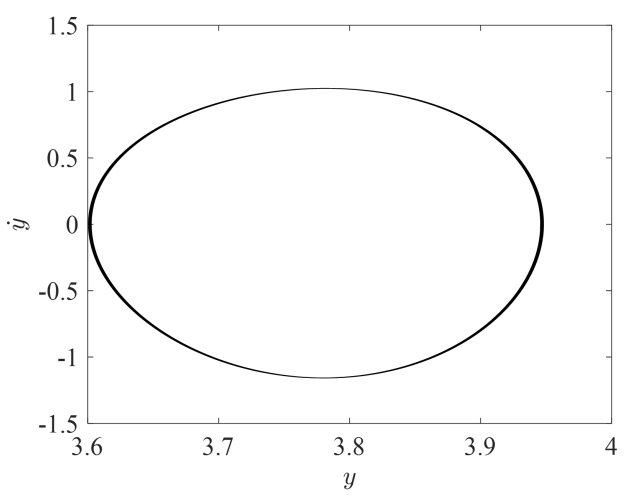

(f) $\omega=2 \pi$

Figure 4. Variation of the system phase diagramwith the rotational angular velocity of the driving wheel

\subsection{Lyapunov exponents}

The largest Lyapunov exponent curve respect to time calculated by above method is showed in Fig.5, in which the figures 5(a) corresponding to the left columns are obtained from the three-link model, and the figures 5(b) corresponding to the right columns are 
obtained by the proposed model. The experiment results show that the direction of the angular velocity has little effect on the Lyapunov exponents. With the increasing of the angular velocity, the Lyapunov exponents obtained from the proposed method become bigger and bigger, this shows that the exponents is sensitive to the initial angular velocity. On the contrary, the Lyapunov exponents obtained from the three-link model are not sensitive to the angular velocity. As the angular velocity is smaller, the exponents are greater than 0; as it becomes larger, the exponents are almost equal to 0 . The results are consistent with the phase diagrams, that is, the three-link system is almost a simple harmonic vibration system, and so it is difficult to identify the cow's health states exactly.
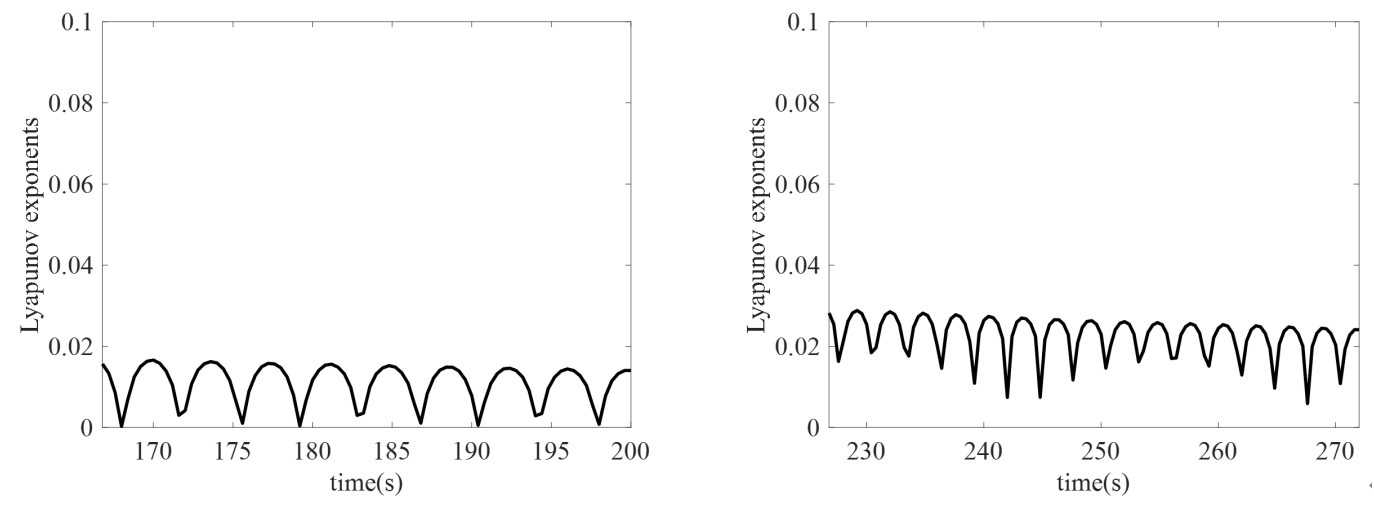

$\omega=2 \pi$
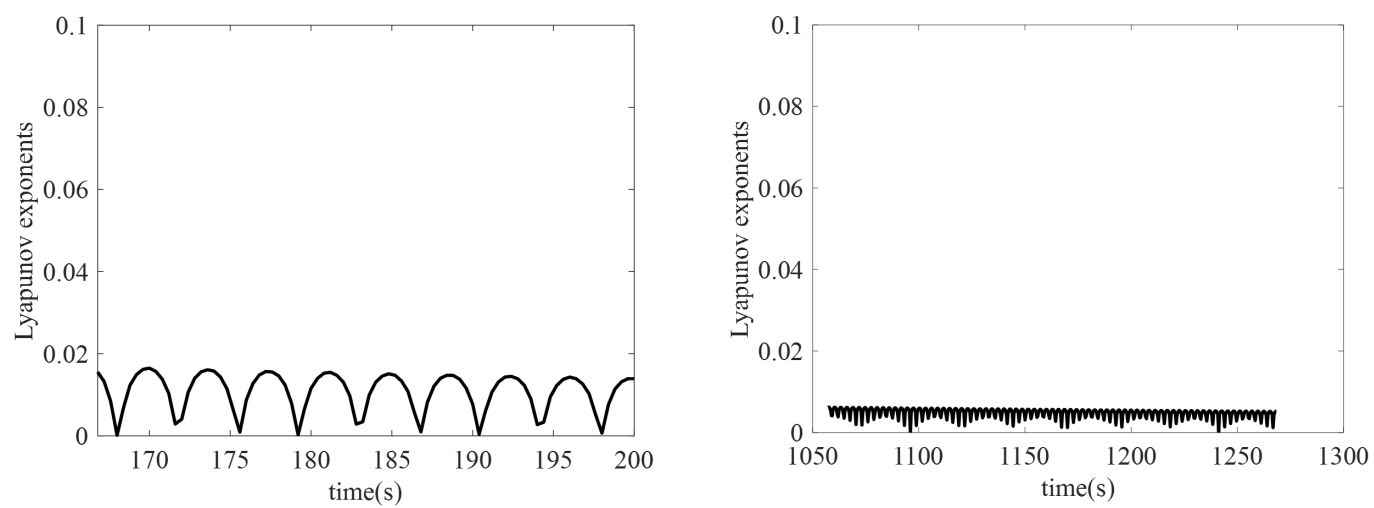

$\omega=-2 \pi$
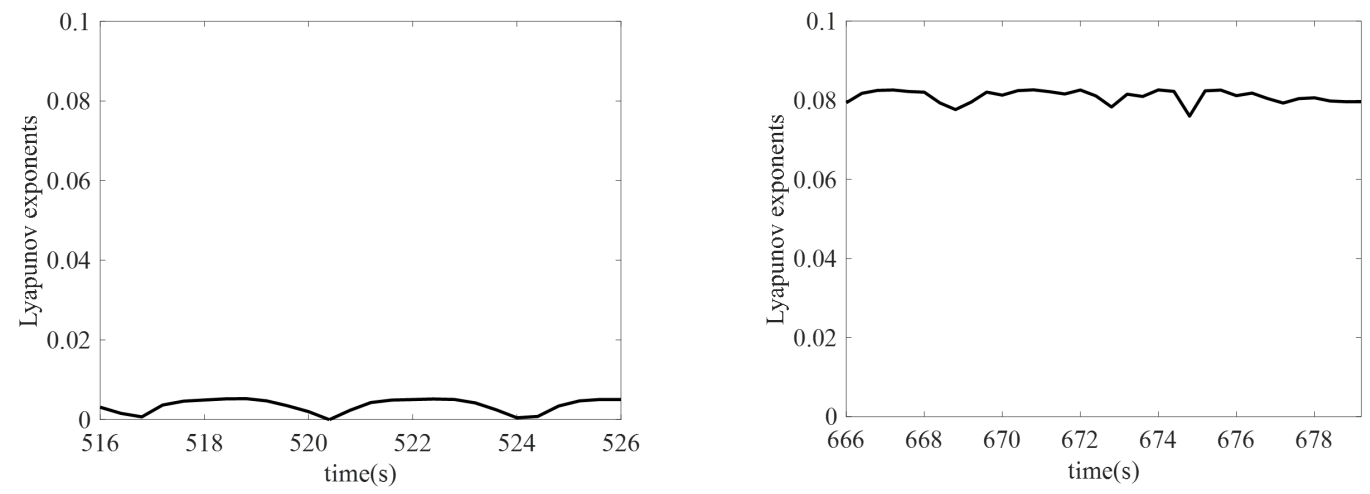

$\omega=6 \pi$ 

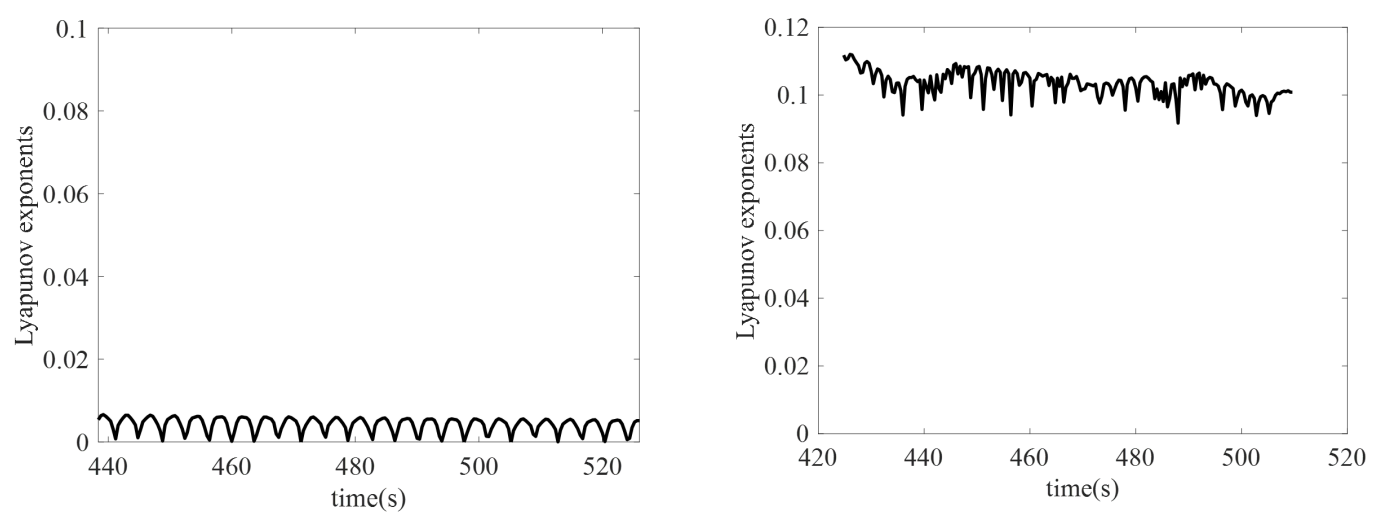

$\omega=-6 \pi$
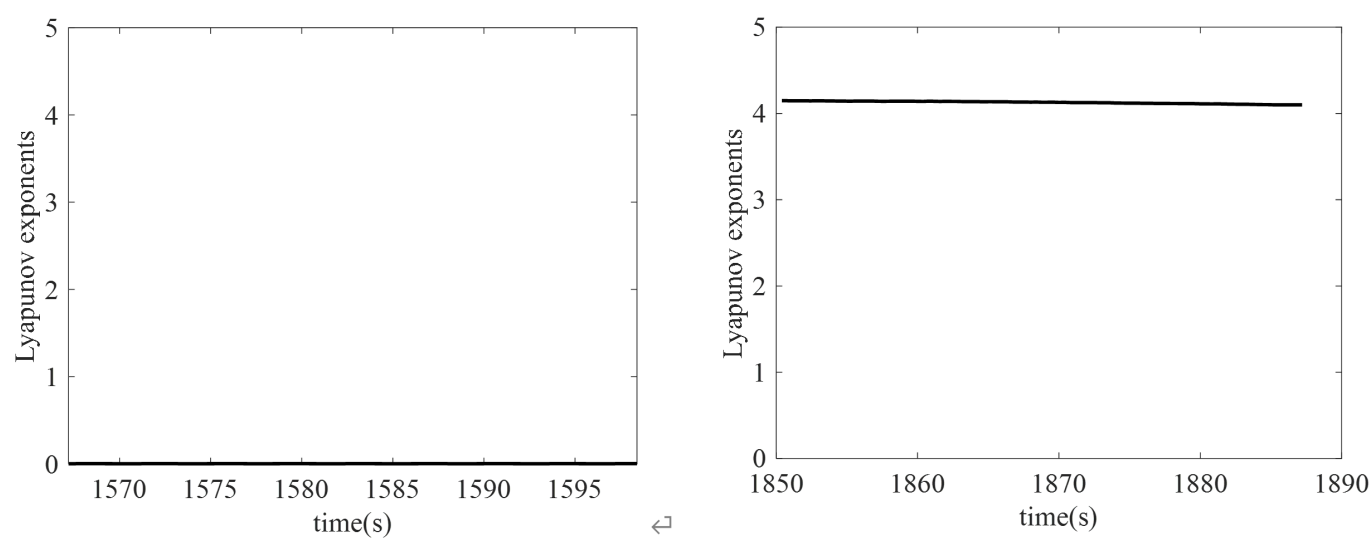

$\omega=20 \pi$
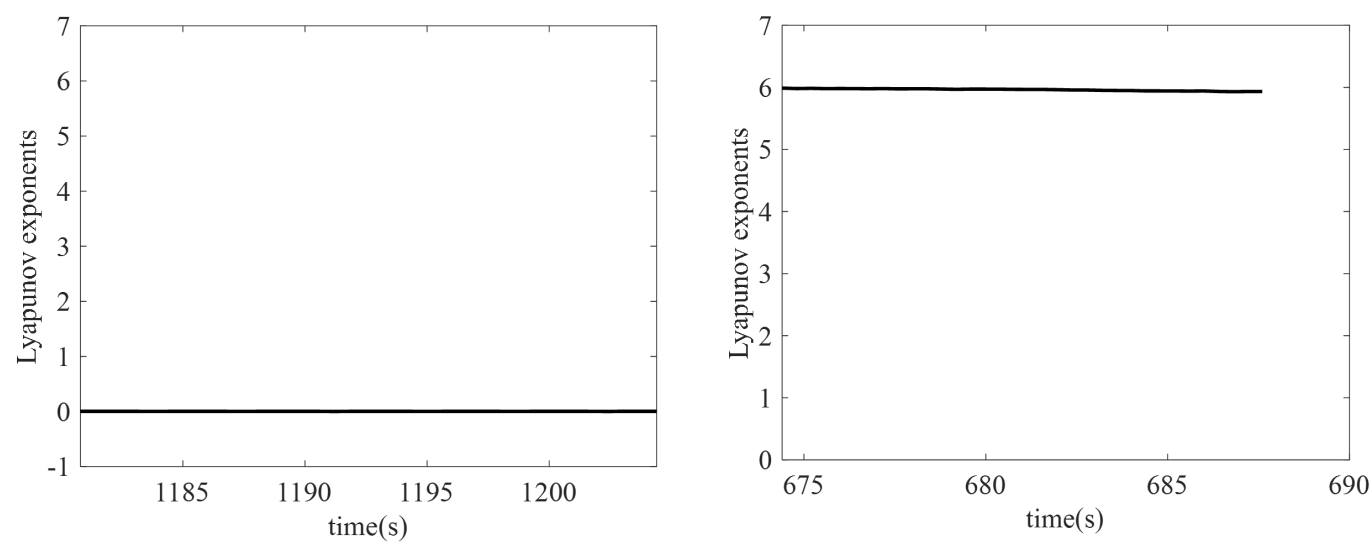

$\omega=-20 \pi$

(a) Three-link system

(b) Proposed model

Figure 5. The largest Lyapunov exponent curve respect to time

\section{Conclusions}

The new chaos diary cow leg dynamic model proposed based on the relationship between period-doubling bifurcation and chaos phenomenon has stronger chaos characteristics and more relaxed parameter selection compared with the three-link chaos vibration system studied before. Eccentric wheel has stable kinematics, small vibration and impact, simple structure and processing technology, and is easy to process and realize. The further work is to use qualitative analysis theory and optimization theory method to analyze the 
global dynamic structure of the system, and find the optimal value of the system parameters based on the comprehensive consideration of the power, mechanical strength and chaotic component spectrum of the system.

Author Contributions: Conceptualization, M.S. and X.R.; methodology, X.R.; software, M.S.; validation, M.S., X.R.; formal analysis, M.S.; investigation, L.M.; resources, X.R.; data curation, M.K.; writing-original draft preparation, X.R.; writing—review and editing, M.S.; visualization, L.M.; supervision, M.S.; project administration, M.S.; funding acquisition, M.S. All authors have read and agreed to the published version of the manuscript.

Funding: This research was funded by Shandong Provincial Natural Science Foundation grant number ZR2020MF019.

Institutional Review Board Statement: Not applicable.

Informed Consent Statement: Not applicable.

Data Availability Statement: Not applicable.

Conflicts of Interest: The authors declare no conflict of interest.

\section{References}

1. J.C.Zaffino Heyerhoff, S.J.LeBlanc, T.J.DeVries, C.G.R. Nash, J.Gibbons, K.Orsel, H.W.Barkema, L.Solano, J.Rushen, A.M.de Passille, and D.B.Haley. Prevalence of and factors associated with hock, knee, and neck injuries on dairy cows in freestall housing in Canada.J.Dairy Sci. 2014, 97, 173-184.

2. Ningning Feng, Xi Kang, Haoyuan Han, Gang Liu, Yan'e Zhang, and Shuli Mei. Research on a Dynamic Algorithm for Cow Weighing Based on an SVM and Empirical Wavelet Transform.Sensors. 2020, 20, 5363,doi:10.3390/s20185363.

3. Andreas Merker, Dieter Kaiser, Andre Seyfarth, and Martin Hermann. Stable running with asymmetric legs: a bifurcation approach.International Journal of Biuration and Chaos. 2015, 25, 1550152.

4. Nolte, D. D. The tangled tale of phase space.Physics Today 2010, 63,33-38.

5. Omar MB, Bingi K, Prusty BR, Ibrahim R. Recent Advances and Applications of Spiral Dynamics Optimization Algorithm: A Review. Fractal and Fractional 2022, 6, 27.https:/ /doi.org/10.3390/fractalfract6

6. Koltun APS, Lenzi EK, Lenzi MK, Zola RS. Diffusion in Heterogenous Media and Sorption-Desorption Processes. Fractal and Fraction 2021, 5, 183.https://doi.org/10.3390/fractalfract5040183

7. Hena Rani Biswas, Md. Maruf Hasan and Shujit Kumar Bala. Chaos theory and its applications in our real life .Barishal University Journal Part 1 2018, 5, 123-140.

8. P.C. Muller. Calculation of Lyapunov exponents for dynamical systems with discontinuities. Solitons Fract. 1995, 5, 1671-1681.

9. Styr, B. , and I. Slutsky. Imbalance between firing homeostasis and synaptic plasticity drives early-phase Alzheimer's disease. Nature Neuroscience 2018, 21, 463-473.

10. Tang K M, Wang Z L, Shi X R. Electrical activity in a time-delay four-variable neuron model under electromagnetic induction. Frontiers in Computational Neuroscience 2017, 11(105), 105-112.

11. Lyu M, Wang C, Ren G.Model of electrical activity in a neuron under magnetic flow effect. Nonlinear Dynamics 2016, 85, 1479-1490.

12. Long Y.; Liang Y. Modren Engineering Dynamics-Random E Chaos, 1rd ed.; Science Press: Beijing, China, 1998; pp. 86-97.

13. Xing R., Yang Y. A New Chaotic Exciter and Its Dynamic Characteristics. Journal of China Agricultural Unviersity 2014, 19(1), 175-179.

14. G. Benettin, L. Galgani, A. Giorgilli, and J. M. Strelcyn. Lyapunov characteristic exponents for smooth dynamical systems: a method for computing all of them. Meccanica 1980, 10, 142-149.

15. R. Benzi, G. Paladin, G. Parisi, and A. Vulpiani, Characterisation of intermittency in chaotic systems Journal of Physics A General Physics 1985, 18, 2157-2165.

16. Jahanzaib LS, Trikha P, Matoog RT, Muhammad S, Al-Ghamdi A, Higazy M. Dual PentaCompound Combination Anti-Synchronization with Analysis and Application to a Novel Fractional Chaotic System. Fractal Fract. 2021, 5(4), 264. https://doi.org/10.3390/fractalfract5040264 